\title{
First drugs found to inhibit elusive cancer target
}

Ever since scientists discovered the cancerpromoting gene MYC in the late 1970s, researchers have dreamt of developing drugs that inhibit its function. Yet efforts to target MYC activity have proven unsuccessful, in part because the protein product encoded by the oncogene lacks an obvious target-binding site. Now, however, scientists from a handful of research groups have found a way to inhibit MYC indirectly-by preventing an upstream protein from instigating the expression of MYC and its downstream targets. Buoyed by the promising therapeutic effects that such experimental drugs have had in mice with several types of cancer, companies are racing to test the molecules in clinical trials, despite lingering questions about how, exactly, they work.

"We, like everyone else, are very excited," says Brian Huntly, a hematologist at the University of Cambridge in the UK. "We're very keen to get this into humans as soon as possible."

In healthy cells, MYC directs important aspects of cell growth and cell cycle progression. But scientists have also found the oncoprotein either mutated or amplified in more than 100 different cancers, including breast and prostate. Like most transcription factors, MYC directly binds DNA and activates protein transcription. But it also adds acetyl marks to lysine residues on chromatin to modify the expression of other genes.

With this in mind, James Bradner, a medical oncologist at the Dana-Farber Cancer Institute in Boston, turned his attention to BRD4, a so-called 'bromodomain' protein that reads these markings on chromatin and activates transcription along surrounding DNA. Bradner suspected that BRD4 might have a role in carcinogenesis, partially because of research implicating it in an aggressive form of cancer called NUT midline carcinoma. BRD4 also interacts with some of the same protein complexes that MYC does ${ }^{1,2}$, and Bradner knew it was possible to inhibit BRD4, because Japan's Mitsubishi Tanabe Pharma Corporation had filed a patent in 2009 for a molecule that did just that.

Bradner and his colleagues devised JQ1, a small molecule based on the structure of the Mitsubishi compound that binds to bromodomains, preventing them from reading acetyl-lysine residues and modulating transcription. Reporting last December, they found that JQ1 caused cancer cells to differentiate and stop growing in a cell line derived from human NUT midline carcinoma tissue and in mouse xenografts with the disease ${ }^{3}$.

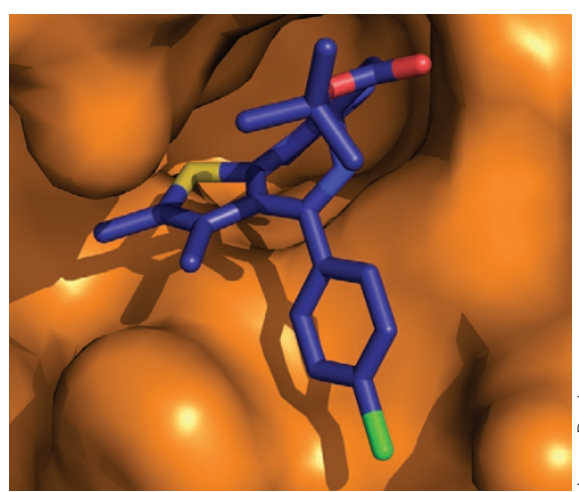

MYC stick: JQ1 binds a pocket of BRD4.

More recently, Bradner's team showed that JQ1 works in part by suppressing the transcription of MYC and its downstream gene targets. What's more, the drug allows mice with multiple myeloma to live $50 \%$ longer than untreated ones, and "the compound was well tolerated even in immunocompromised animals," says Bradner, who published the findings in September ${ }^{4}$. However, the drug's half life is only one hour, so it has short-lasting effects.

\section{Overwhelming response}

It's not just myeloma where BRD4 inhibitors like JQ1 have potential; they seem to slow the progression of mouse leukemia and lymphoma, too. In work published online in August, a team from the Cold Spring Harbor Laboratory in Long Island, New York developed an RNA interference screen to identify chromatin modifiers that might play a part in acute myeloid leukemia (AML). Soon after identifying BRD4 as a key hit, the researchers saw Bradner's 2010 paper describing JQ1 and asked to work with it.

"We knew we had something big on our hands when we treated the first mice," says study lead Johannes Zuber, now at the Research Institute of Molecular Pathology in Vienna. "The scale of the effect was overwhelming," with JQ1 extending the life spans of the mice by more than $20 \%$ on average ${ }^{5}$. And, like Bradner, Zuber identified MYC as one of the main genes JQ1 suppressed.

Further evidence of JQ1's potential came last month when scientists at Constellation Pharmaceuticals in Cambridge, Massachusetts reported that JQ1 slowed tumor growth in mouse models of Burkitt's lymphoma and AML, but not in a breast cancer cell line ${ }^{6}$. Although this validates the strategy of targeting bromodomains to halt the growth of blood cancers, these findings suggest that "it's probably not going to be quite as helpful for solid-organ malignancies," Huntly says.

But MYC doesn't seem to be the whole story. "There are cells in which MYC is affected by the compound, but the cells continue to proliferate," says Kevin Lee, head of the EpiNova Discovery Performance Unit at GlaxoSmithKline (GSK) in Stevenage, UK. In October, Lee, together with Huntly and others, showed that two BRD4 inhibitors developed by GSK lengthened the lifespan of mice with mixed-lineage fusion leukemia, another rare cancer characterized by chromosomal translocations. But, in addition to $M Y C$, the GSK drugs also reduced expression of $B C L 2$ and $C D K 6$, two genes that have also been implicated in leukemia ${ }^{7}$.

"Whilst MYC is a big player, I think it's going to be only one of a number of very important of downstream transcriptional targets for these [bromodomain] inhibitors," Huntly says.

The big question now is whether bromodomain blockers turn out to be workable drugs. Bradner, for one, is betting on it, having recently launched a company called Tensha Therapeutics to develop them further. In September, the Cambridge, Massachusettsbased startup announced that it had secured \$15 million from HealthCare Ventures to develop drug-like derivatives of JQ1. According to Lee, GSK is also looking into developing a range of different bromodomain inhibitors in its library; Mitsubishi Tanabe, meanwhile, has none in its pipeline, according to corporate communications manager Kazuko Hamada.

Ultimately, "the proof of the pudding is really going to come when people look a little more carefully at off-target effects," says Edward Prochownik, director of oncology at the Children's Hospital of Pittsburgh, noting that bromodomain proteins probably have important functions in healthy cells, too. Nevertheless, these first MYC inhibitors are creating quite a buzz in the cancer community-and Bradner suspects this is just the beginning.

"We provided JQ1 to over 120 academic, industrial and governmental labs," he says. "The first wave of research papers is just starting to appear."

Melinda Wenner Moyer

1. Bisgrove, D.A., Mahmoudi, T., Henklein, P. \& Verdin, E. Proc. Natl. Acad. Sci. USA 104, 13690-13695 (2007).

2. Rahl, P.B. et al. Cell 141, 432-445 (2010).

3. Filippakopoulos, P. et al. Nature 468, 1067-1073 (2010).

4. Delmore, J.E. et al. Cell 146, 904-917 (2011)

5. Zuber, J. et al. Nature 478, 524-528 (2011).

6. Mertz, J.A. et al. Proc. Natl. Acad. Sci. USA 108, 16669-16674 (2011).

7. Dawson, M.A. et al. Nature 478, 529-533 (2011). 